\title{
Cognitive behavioral therapy improves adherence to antiretroviral therapy in HIV-infected patients: a prospective randomized controlled trial from eastern India
}

\author{
Soumak Chattopadhyay ${ }^{1}$, Somedeb Ball ${ }^{1}$, Arindam Kargupta ${ }^{1}$, Payel Talukdar ${ }^{2}$, Krishnendu Roy ${ }^{1}$, \\ Arunansu Talukdar ${ }^{1}$, Prathama Guha ${ }^{2}$
}

${ }^{1}$ Medical College and Hospital, Kolkata, India

${ }^{2}$ NRS Medical College \& Hospital, Kolkata, India

\begin{abstract}
We know that human immunodeficiency virus (HIV) is an important pandemic of modern generation causing large death toll worldwide. India is also no exception with 2.12 million people already affected by HIV. The only way to reduce morbidity and mortality due to HIV is antiretroviral therapy (ART). But a high degree of adherence is critical for the effectiveness of ART, and non-adherence is associated with an overall poor outcome in HIV patients. We conducted a randomized controlled prospective study to find out the factors responsible for non-adherence to ART and to assess the role of cognitive behavioral therapy (CBT) to improve adherence. In this study, 60 HIV/AIDS patients with non-adherence $(<95 \%$ adherence) were randomized into two groups consisting of 30 patients each, one group (intervention) was administered structured CBT and the other group (control) was put on standard therapy. Factors responsible for non-adherence were overconcern about side effects (33\%), polarized thinking (27\%), mind reading (27\%), and magnification (20\%). Mean adherence was $100 \%$ in the intervention arm vs. $97.5 \%$ in controls $(p=0.00)$, Beck's depression score was reduced significantly in the intervention group ( $p=0.00$ ), but no significant difference was noted in CD4 count $(p=0.37)$. We strongly recommend inclusion of CBT in the routine management protocol for HIV/AIDS.
\end{abstract}

HIV AIDS Rev 2017; 16, 2: 89-95 DOI: https://doi.org/10.5114/hivar.2017.67303

Key words: AIDS, antiretroviral therapy, adherence to ART, cognitive behavioral therapy in HIV, Beck's depression score.

\section{Introduction}

Human immunodeficiency virus (HIV) has been a difficult pandemic to control ever since its inception. According to the latest data of UNAIDS (Joint United Nations Program on HIV/AIDS), globally about 36.9 million people are harboring HIV infection, and about 1.2 million people die of it every year [1]. In India, the total number of patients who are living with HIV (PLHIV) currently is about 2.12 million, and around 67.6 thousand patients died last year due to HIV-related causes. Various control measures, including administration of antiretroviral therapy (ART), have led to a reduction in the prevalence of HIV among adults in India from $0.34 \%$ in 2007 to $0.26 \%$ in 2015 according to latest NACO (National AIDS Control Organization) data [2].
Address for correspondence: Dr. Arindam Kargupta, Medical College and Hospital, 88 college street, 700073 Kolkata, India, phone: 00918697180608 ,

e-mail: drarindamkg89@gmail.com
Article history:

Received: 26.08 .2016

Received in revised form: 08.12.2016

Accepted: 11.12.2016

Available online: 21.04 .2017
International Journal of HIV-Related Problems

HIV \& AIDS

R e v i e w 
Presently there is no permanent cure available for HIV, and the only effective therapy available is ART. These drugs target various steps in the life cycle of HIV and on continuous intake, they cause profound suppression of the HIVRNA load in the body, leading to a boost in patient's immunity. Anti-retroviral therapy also causes a reduction in the occurrence of HIV-related infections and malignancies. One of the main limitations of the therapy in HIV is that once started, ART has to be taken for the rest of the life. To ensure the effective suppression of HIV-RNA load, these drugs need to be taken with an adherence level preferably of $>95 \%$. Even a short discontinuation in the form of drug holiday could increase the viral load to a huge extent, and thus may lead to ART failure, the emergence of resistant viral strains, quicker progression to AIDS, more frequent hospitalizations and increased overall mortality $[3,4]$. In fact, studies have shown that in patients with an inadequate adherence level, even an improvement of $10 \%$ in adherence can bring about favorable biological outcomes [5].

Cognitive behavioral therapy (CBT), a form of psychotherapy, is a focused approach to the treatment of emotional, behavioral and psychiatric ailments. A cognitive approach is necessary for changing the pattern of thinking whereas behavioral therapy focuses on the associated action. Cognitive behavioral therapy has been tried as an adjunct to available treatment modalities in different disease conditions. Thus, a comprehensive CBT protocol could help increase the drug adherence in the HIV patients as well. To date, there have been many studies that assessed CBT as a part of the treatment protocol in different groups of HIV patients. Very few of them have looked into the effect of CBT on adherence to ART in patients who did not have documented depression at baseline [6,7]. Recent studies have linked cognitive-affective vulnerabilities with perceived barriers to medication adherence in HIV patients [8]. In this backdrop, this study was undertaken in a tertiary-care hospital of Kolkata, the largest metropolitan city in eastern India, with an objective of finding out the factors behind the poor adherence to ART and also to evaluate the impact of CBT on the adherence among the HIV-positive patients.

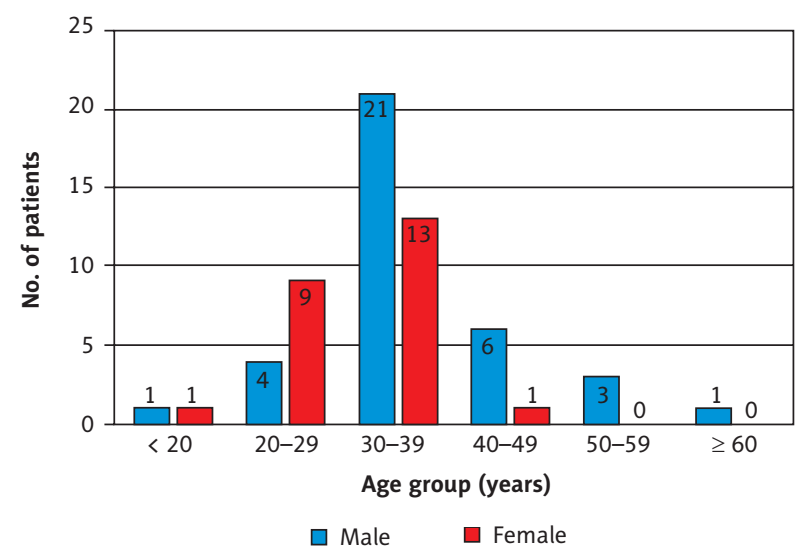

Figure 1. Age distribution of study subjects

\section{Material and methods}

\section{Study design}

This randomized prospective controlled study was conducted in the HIV referral center of the Medical College and Hospital, a tertiary-care hospital in eastern India. The study was interventional in nature and longitudinal in the mode of data collection. An approval for the study was obtained from the institutional ethics committee. All cases of HIV attending the referral center between August 2013 and July 2014 were screened. The HIV patients of both sexes, aged above 18 years and below 65 years were selected. All these patients in our study were on ART, with a baseline adherence level of less than 95\% (measured by a treatment card and supported by patient recall). Patients with active substance abuse, those with psychosis at present or mental retardation, generalized debilitated patients, and those who did not give consent, were excluded from the study.

Patients fulfilling the inclusion criteria were randomized according to the block randomization schedule. A total of 60 patients were randomized into two groups, consisting of 30 patients each. Patients in the intervention group were administered a structured CBT and those in the control group received only psychoeducation and supportive counselling.

Informed consent was taken from the study participants after explaining the importance of the study to them in detail. Anonymity and confidentiality were assured to all the participants. Participants were blind to the group (intervention or control) they belonged. The primary objective of the study was to enumerate the factors responsible for non-adherence to ART among HIV-positive patients and to assess whether CBT helps to improve the adherence in these patients. Secondary objectives were also to study the effect of CBT on the depression score (Beck's Depression Inventory) and the CD4 count of patients.

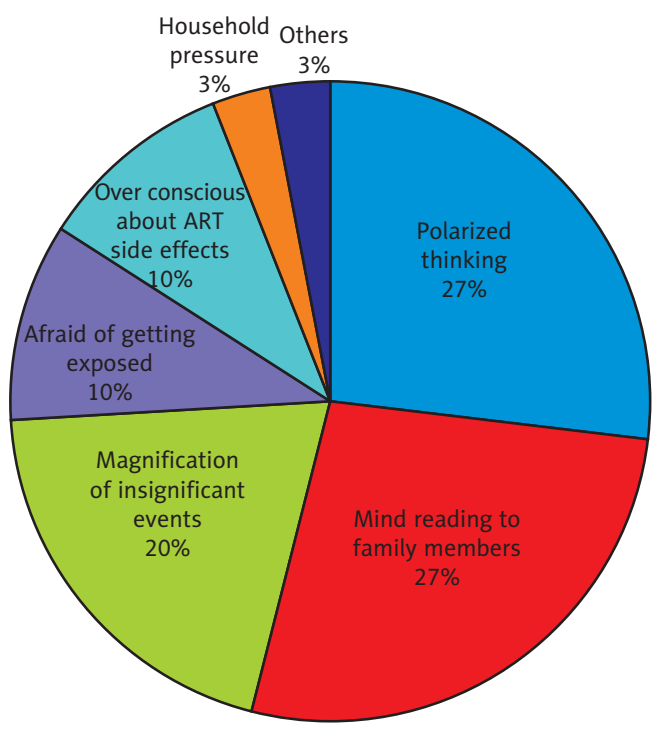

Figure 2. Common factors for non-adherence 
Table 1. Intervention - CBT protocol

\begin{tabular}{|c|c|c|c|}
\hline Module no. & $\begin{array}{c}\text { No. of } \\
\text { sessions }\end{array}$ & Module topic & Content \\
\hline 1 & 2 (two) & Adherence training & $\begin{array}{l}\text { Consent of individual patient taken in written format } \\
\text { Depression level assessed by "Beck's depression inventory" } \\
\text { Psychoeducation about medicine resistance } \\
\text { Motivational interview }\end{array}$ \\
\hline 2 & 1 (one) & $\begin{array}{l}\text { Behavioral } \\
\text { activation }\end{array}$ & $\begin{array}{l}\text { Interviewing the patient about daily routine } \\
\text { Associated mood charts } \\
\text { Bringing about realistic, structured behavioral changes in small steps }\end{array}$ \\
\hline 3 & 3 (three) & $\begin{array}{l}\text { Cognitive } \\
\text { reconstructive }\end{array}$ & $\begin{array}{l}\text { Interviewing for negative automatic thought or core beliefs with specific } \\
\text { examples like: } \\
\text { - Mind reading } \\
\text { - Magnification } \\
\text { - Black \& white thinking etc. } \\
\text { Other commonly expressed concerns related to poor adherence were: } \\
\text { - Fear of getting exposed to other family members or neighbors } \\
\text { - Concern of side effects of drugs } \\
\text { - Child pressure \& other household chore pressure } \\
\text { Automatic thought records were used by patients with some help from therapist } \\
\text { The cognitive errors were corrected using individual cognitive strategies }\end{array}$ \\
\hline 4 & 1 (one) & Problem solving & $\begin{array}{l}\text { Intention } \\
\text { Define problem } \\
\text { Evaluation of alternatives } \\
\text { Decision making } \\
\text { Implementation of solution }\end{array}$ \\
\hline 5. & 1 (one) & Relaxation training & $\begin{array}{l}\text { Progressive muscle relaxation } \\
\text { Diaphragmatic breathing }\end{array}$ \\
\hline
\end{tabular}

\section{CBT protocol}

The protocol for CBT was designed in consultation with the experts in the Department of Psychiatry of the Institute. Cognitive behavioral therapy was administered in a group therapy format. The protocol was then administered to the intervention group (in two groups over a period of six months, eight sessions, each of 30 minutes' length). Cognitive behavioral therapy was administered in five modules - module 1 was about adherence training, consisting of two sessions, module 2 was about behavioral activation (one session), module 3 - cognitive reconstruction (three sessions), module 4 - problem solving (one session), and module 5 - relaxation training (one session). Details of the protocol have been summarized in Table 1.

\section{Beck's Depression Inventory}

Beck's depression inventory (BDI) is a self-report rating inventory containing 21 questions used for measuring the characteristic attitude and symptoms of depression. Here each answer is evaluated on a scale of 0 to 3 , and the higher the score the greater the severity of depression. BDI score of less than 10 is not considered as clinically significant depression $[9,10]$. However, it can provide a quantitative assessment of the intensity of depression and also helps to monitor the response to therapy, it has a problem of being excessively patient dependent. In our study, BDI was used in patients at the baseline and at the end to assess the severity of depressive symptoms in them.

\section{Data collection}

A pre-designed and pre-tested schedule was selected for our study, keeping in mind the objectives of the study. It had three parts - the first part for collecting information about the demographic and socio-economic characteristics of the patients; the second part included questions from the Beck's Depression Inventory (BDI) for measuring the depression score of the participants, and the third part included information about the clinical and laboratory findings of the patients. The CD4 lymphocyte count was measured in patients at the baseline and the end of the study. A blood sample was collected following the standard aseptic procedure. Prior treatment history was taken for measuring the level of adherence (adherence = drugs consumed/drugs given $\times 100$ ) in patients. Body weights of the participants were measured following the standard WHO (World Health Organization) guideline.

\section{Statistical analysis}

PSPP and R, both open source statistical analytical software, were used for data analysis. All data were entered into 
Table 2. Baseline characteristics of the study participants $(n=60)$

\begin{tabular}{l|c|c|c}
\hline Baseline characteristics & $\begin{array}{c}\text { Intervention } \\
n=30 \\
\text { Mean (SD) }\end{array}$ & $\begin{array}{c}\text { Control } \\
n=30 \\
\text { Mean (SD) }\end{array}$ & $p$-value \\
\hline Age (years) & $34.60(6.55)$ & $33.57(8.71)$ & 0.61 \\
\hline Distance from the treatment center (km) & $23.27(14.38)$ & $26.00(22.58)$ & 0.58 \\
\hline Duration of education (years) & $3.27(2.96)$ & $4.90(3.81)$ & 0.07 \\
\hline Total family income (rupees) & $5000.00(2406.89)$ & $2983.33(1759.13)$ & 0.00 \\
\hline Beck's Depression Score before CBT & $20.53(9.71)$ & $21.33(9.45)$ & 0.75 \\
\hline Adherence before CBT & $89.31(2.97)$ & $88.82(5.10)$ & 0.65 \\
\hline CD4 count before CBT & $375.13(155.18)$ & $327.60(164.75)$ & 0.26 \\
\hline Body weight before CBT (in kg) & $54.27(5.61)$ & $50.50(7.74)$ & 0.04 \\
\hline
\end{tabular}

SD - standard deviation, CBT - Cognitive Behavioral Therapy

$p<0.05$ were considered significant; significant findings were marked in bold.

$p$-value were calculated using independent sample t-test; variance was calculated using Levene's test.

Microsoft Excel, the spreadsheet program. After entering the data, it was checked for any duplicate or erroneous entry. The spreadsheet file was saved into comma separated value format. This file was then imported into PSPP, the open source statistical analytical software. Variables were recorded and labeled into different variables, required for appropriate statistical analysis. For categorical risk factors, contingency tables were used, and the strength of association of their occurrence in the two groups was measured using chi-square test. Continuous variables (e.g. age, Beck's depression score) were expressed as the mean and standard deviation (SD). Independent $t$-test was used to check whether any statistically significant difference existed between the two groups - intervention and control.

\section{Results}

In our study, both groups (intervention and control) were comparable on various baseline characteristics such as age, sex, education, residential status, literacy level, distance from the treatment center, etc. (Table 2). There was no statistically significant difference in baseline CD4 count $(p=0.26)$, adherence status ( $p=0.65)$ as well as Beck's depression score ( $p=0.75$ ) between the two groups. The study participants were mostly males (60\%) and in the 30-39-year age group (56.67\%) (Figure 1). The majority of them had an active sexual partner (85\%). Study participants lived mostly in rural areas (55\%). Most of these patients (70\%) did not have any preexisting comorbidities. A total number of drop out at the end of four months was 3 (i.e. 5\%), two of them in the intervention group and one in the control group and rest of the patients continued until the end of the study.

The factors of interest for non-adherence were polarized thinking (or black and white thinking), which was present in $27 \%$ of patients; $20 \%$ of patients had magnification characterized by exaggeration of importance of insignificant events; $27 \%$ of patients were found to have mind reading to other family members as if they are blaming or criticizing him/her; while $10 \%$ were afraid of getting exposed to the family members or neighbors; $10 \%$ of the patients were over conscious about the side effects of ART. A minority (3\%) of study population stated that child pressure or household pressure was responsible for non-adherence. In many instances, a single patient stated more than one factors for their non-adherence. Among the factors, magnification, mind reading, and polarization are common cognitive errors, which we addressed in our CBT sessions. Common factors for non-adherence have been depicted in Figure 2.

Before CBT, the mean adherence in the intervention group was $89.31 \%(\mathrm{SD}=2.97 \%)$ and in the controls, it was $88.82 \%(\mathrm{SD}=5.10 \%)$ with not statistically significant difference $(p=0.65)$. Mean Beck's depression score in the intervention group was $20.53(\mathrm{SD}=9.71)$ and in the control group, 21.33 ( $\mathrm{SD}=9.45$ ), this difference was not statistically significant either $(p=0.75)$. After CBT had been administered, a significant difference was observed between the two groups. All the patients in the intervention arm showed a mean adherence to ART of $100 \%$ (vs. $97.51 \%$ in controls, $p=0.00$ ), thus reflecting the effectiveness of CBT in improving adherence. Following CBT sessions, Beck's depression score also dropped significantly in the intervention group, with a mean of 15.40 ( $\mathrm{SD}=7.00)$, as opposed to a mean of 23.37 ( $\mathrm{SD}=10.14)$ in controls $(p=0.00)$. Body weight (in $\mathrm{kg}$ ) of patients also showed a statistically significant improvement in the intervention group (mean 56.20, SD $=6.85$ ), compared to controls (mean 49.30, $\mathrm{SD}=8.57)(p=0.00)$. However, CD4 count did not show any statistically significant difference between two groups even after CBT sessions $(p=0.37)$. Principal outcome characteristics of our study have been summarized in Table 3.

\section{Discussion}

Studies have conclusively shown that adherence is the key to effective therapy in HIV, and poor adherence has 
Table 3. Outcome characteristics of the study participants $(n=57)$

\begin{tabular}{l|c|c|c}
\hline Baseline characteristics & $\begin{array}{c}\text { Intervention } \\
n=28 \\
\text { Mean (SD) }\end{array}$ & $\begin{array}{c}\text { Control } \\
n=29 \\
\text { Mean (SD) }\end{array}$ & $p$-value \\
\hline Beck's Depression Score after CBT & $15.40(7.00)$ & $23.37(10.14)$ & 0.00 \\
\hline Adherence after CBT & $100.00(0.00)$ & $97.51(3.03)$ & 0.00 \\
\hline CD4 count after CBT & $430.27(145.76)$ & $392.63(172.20)$ & 0.37 \\
\hline Body weight after CBT (in kg) & $56.20(6.85)$ & $49.30(8.57)$ & 0.00 \\
\hline
\end{tabular}

CBT-Cognitive Behavioral Therapy

$p<0.05$ were considered significant; significant findings were marked in bold.

$p$-value were calculated using independent sample t-test; variance was calculated using Levene's test.

Table 4. Relationship of the baseline characteristics with outcome variables

\begin{tabular}{|c|c|c|c|c|c|c|c|c|c|}
\hline & & Age & $\begin{array}{c}\text { Distance from } \\
\text { the treatment } \\
\text { center }(\mathrm{km})\end{array}$ & $\begin{array}{l}\text { Duration of } \\
\text { education } \\
\text { (years) }\end{array}$ & $\begin{array}{c}\text { Total } \\
\text { family } \\
\text { income } \\
\text { (rupee) }\end{array}$ & $\begin{array}{c}\text { Beck's } \\
\text { Depression } \\
\text { Score after } \\
\text { CBT }\end{array}$ & $\begin{array}{l}\text { Adherence } \\
\text { after CBT }\end{array}$ & $\begin{array}{l}\text { CD4 } \\
\text { count } \\
\text { after } \\
\text { CBT }\end{array}$ & $\begin{array}{c}\text { Body } \\
\text { weight } \\
\text { after } \\
\text { CBT } \\
\text { (kg) }\end{array}$ \\
\hline \multirow{2}{*}{ Age (years) } & $r$ & 1.00 & & & & & & & \\
\hline & $p$-value & & & & & & & & \\
\hline \multirow{2}{*}{$\begin{array}{l}\text { Distance from the } \\
\text { treatment center }(\mathrm{km})\end{array}$} & $r$ & -0.04 & 1.00 & & & & & & \\
\hline & $p$-value & 0.79 & & & & & & & \\
\hline \multirow{2}{*}{$\begin{array}{l}\text { Duration of } \\
\text { education (years) }\end{array}$} & $r$ & -0.35 & 0.34 & 1.00 & & & & & \\
\hline & $p$-value & 0.01 & 0.01 & & & & & & \\
\hline \multirow{2}{*}{$\begin{array}{l}\text { Total family income } \\
\text { (rupee) }\end{array}$} & $r$ & 0.06 & -0.07 & 0.22 & 1.00 & & & & \\
\hline & $p$-value & 0.67 & 0.59 & 0.09 & & & & & \\
\hline \multirow{2}{*}{$\begin{array}{l}\text { Beck's Depression } \\
\text { Score after CBT }\end{array}$} & $r$ & -0.10 & 0.32 & 0.18 & -0.30 & 1.00 & & & \\
\hline & $p$-value & 0.42 & 0.01 & 0.16 & 0.019 & & & & \\
\hline \multirow{2}{*}{ Adherence after CBT } & $r$ & -0.07 & -0.02 & -0.17 & 0.16 & -0.19 & 1.00 & & \\
\hline & $p$-value & 0.58 & 0.91 & 0.21 & 0.228 & 0.153 & & & \\
\hline \multirow{2}{*}{ CD4 count after CBT } & $r$ & -0.31 & -0.18 & 0.08 & 0.22 & -0.15 & 0.02 & 1.00 & \\
\hline & $p$-value & 0.02 & 0.18 & 0.57 & 0.086 & 0.242 & 0.885 & & \\
\hline \multirow{2}{*}{$\begin{array}{l}\text { Body weight after } \\
\text { CBT (kg) }\end{array}$} & $r$ & 0.40 & -0.08 & -0.03 & 0.36 & -0.27 & 0.20 & -0.03 & 1.00 \\
\hline & $p$-value & 0.00 & 0.525 & 0.78 & 0.004 & 0.038 & 0.123 & 0.795 & \\
\hline
\end{tabular}

CBT - Cognitive Behavioral Therapy

Relationship between the variables were tested using Pearson's correlation coefficient ( $r$ ). $p<0.05$ were considered significant and significant findings were marked in bold.

been associated with worse clinical as well as immunological outcomes [3, 4, 11-13]. Poor adherence can also result in the development of viral mutations, which contribute to the development of drug resistance $[14,15]$. Cognitive behavioral therapy is a form of psychotherapy, which is flexible, individualized and can be adapted to a wide range of individuals and a variety of settings. Worldwide, it has been successfully applied in various psychiatric ailments like depression, anxiety, obsessive-compulsive disorder, phobias and eating disorders [16-18]. It has also been used as an adjunct to the existing therapy in various chronic diseases (e.g. migraine, heart failure, etc.) $[19,20]$. Use of CBT can dispel misconceptions and individual cognitive errors about the continuation of ART among HIV patients and thus can help in increasing the adherence to therapy in them. The behavioral aspect of discontinuing the therapy because of feeling well initially could be effectively handled by the behavioral segment of CBT [21].

Several studies over the years have tried to assess the role of CBT in the treatment of HIV patients in various settings $[22,23]$. In a study of adherence to therapy in HIV-positive individuals, five distinct subgroups were identified, with variations noted over the course of the 4-week study. Some additional comparisons of different demographic and behavioral variables found that patients with worst adherence were also having higher rates of substance use. It was also 
noted that the subgroup with a higher degree of cognitive impairment had a consistent drop in the adherence level. These results suggest that there are distinct subgroups of adherers with different behavioral features and interventions are to be designed accordingly to accommodate this variability in behavior [24]. Johnson et al. studied the effect of CBT on adherence in $204 \mathrm{HIV}$-infected patients with self-reported adherence $<85 \%$ at baseline and followed them up for up to 25 months. It was observed that at five months, there was approximately $13 \%$ greater improvement in adherence in the intervention group $(p<0.01)$. At ten months there was still a significant greater adherence in the intervention group, which finally though was not observed at 20 and 25 months follow-up [6]. In another prospective randomized trial comprising of $60 \mathrm{HIV}$ patients, the proportion of patients with an adherence level $>95 \%$ was $70 \%$ in the intervention (CBT) group compared to $50 \%$ in controls $(p=0.014)$ [7]. Berger et al. concluded in their study involving $104 \mathrm{HIV}$-infected persons taking ART that CBSM (Cognitive Behavioral Stress Management) training did not improve the clinical outcome but had lasting effects on quality of life and psychological well-being in patients [25]. In a meta-analysis consisting of 19 studies (1839 participants), it was noted that the participants in the psychosocial intervention arm were more likely to achieve $95 \%$ adherence (OR $1.50 ; 1.16-1.94$ ) than those in the control arm; the effect was also equally significant for an undetectable viral load (OR 1.25; 0.99-1.59). The intervention effect for $95 \%$ adherence was significantly stronger in studies that used recall periods of 2 or 4 weeks (vs. $<7$ days) [26]. Taken together, all the evidence suggests that various ART adherence intervention strategies can be successful, but more research is needed to identify the most efficacious intervention [27].

Recently several studies have looked at the effectiveness of CBT in the improvement of adherence in HIV patients, with or without concomitant depression. Recently published studies from Africa reported better maintenance of adherence with the use of the cognitive behavioral intervention $[28,29]$. A study even highlighted the role of CBT in reducing the intensity of troublesome side effects (fatigue and nausea), noted in patients on ART for a long duration, thus indicating towards a possible role of CBT in potentiation of individual's ability to be able to continue with ART [30].

In our study, we included HIV patients on ART with an adherence level of $<95 \%$. Sixty such patients were selected according to inclusion criteria, and they were divided into two groups. The intervention group $(n=30)$ received eight sessions of CBT over a period of six months according to the protocol, whereas the control group received only psychoeducation and supportive counseling. Both groups continued to receive the ART. At the start of the study, both groups were comparable in their baseline characteristics. The study showed a significantly better mean adherence level and Beck's depression score in patients of the intervention group. The beneficial effect of CBT observed in our study actually reflected the outcome of various international studies undertaken so far $[28,29,31-33]$. But CD4 count did not show any significant difference between 2 groups after CBT sessions as opposed to the finding in some of the recent studies $[32,33]$. When we looked at various factors responsible for non-adherence to ART, polarized thinking (27\%), mind reading (27\%) and magnification (20\%) were found to be the major ones and in fact, some of the patients were having more than one factor together. In polarized thinking all the things are taken as either black or white, which means either they are perfect or a complete failure. As no such complete cure for HIV is available till now and ART intake is associated with multiple adverse effects and risk of drug resistance, patients who have polarized thinking often refuse to continue taking ART. Good clinical response to ART is possible only when the adherence level is $>95 \%$. Thus this type of thought process in patients poses a difficult hurdle to maintain good adherence to ART.

One of major limitations of our study was dropout in the study population. Two patients assigned to the intervention group refused to continue their participation further in the study even after their initial consent, and one in the control group lost to follow-up. This resulted in an increase in the possibility of 'beta' error while assessing the results of the study. Though interestingly there was no dropout in the intervention group after four months of CBT sessions, suggesting that change in perception and mood in patients probably may have played a role in their compliance to the treatment protocol. Also, a follow-up of longer duration than has been done in this study could be helpful in fetching more relevant information about the sustainability of observed outcomes.

\section{Conclusions}

HIV/AIDS being a lifestyle disorder is always a great threat to a country like India because of its huge population. Though this disease is incurable at present, regular and continued consumption of ART can improve the quality of life and overall survival in patients. ART has, in fact, so far been successful in controlling the epidemic but adherence remains a bothersome issue. Our study showed conclusively that a properly structured CBT regime could significantly improve the adherence level to ART. We strongly recommend that public health professionals in India should now seriously consider inclusion of CBT in the management protocol of HIV, and we believe that this may usher new rays of hope in the lives of innumerable HIV-infected individuals.

\section{Conflict of interest}

The authors declared no potential conflicts of interest with respect to the research, authorship, and/or publication of this article.

\section{References}

1. UNAIDS (Joint United Nations Program on HIV/AIDS); http:// www.unaids.org/en/resources/campaigns/HowAIDSchangedeverything/factsheet. 
2. India HIV Estimations 2015 - Technical report; http://www.naco. gov.in/upload/2015\%20MSLNS/HSS/India\%20HIV\%20Estimations\%202015.pdf.

3. Gill CJ, Hamer DH, Simon JL, et al. No room for complacency about adherence to antiretroviral therapy in sub-Saharan Africa. AIDS 2005; 19: 1243-1249.

4. Garcia de Olalla P, Knobel H, Carmona A, et al. Impact of adherence and highly active antiretroviral therapy on survival in HIV-infected patients. J Acquir Immune Defic Syndr 2002; 30: 105-110.

5. Liu H, Miller LG, Golin CE, et al. Repeated measures longitudinal analyses of HIV virologic response as a function of percent adherence, dose timing, genotypic sensitivity, and other factors. J Acquir Immune Defic Syndr 2006; 41: 315-322.

6. Johnson MO, Charlebois E, Morin SF, et al., and the National Institute of Mental Health Healthy Living Project Team. Effects of a Behavioral Intervention on Antiretroviral Medication Adherence Among People Living with HIV. J Acquir Immune Defic Syndr 2007; 46: 574-580.

7. Weber R, Christen L, Christen S, et al.; Swiss HIV Cohort Study. Effect of individual cognitive behavior intervention on adherence to antiretroviral therapy: prospective randomized trial. Antivir Ther 2004; 9: 85-95.

8. Leyro TM, Vujanovic AA, Bonn-Miller MO. Examining associations between cognitive-affective vulnerability and HIV symptom severity, perceived barriers to treatment adherence, and viral load among HIV-positive adults. Int J Behav Med 2015; 22: 139-148.

9. Beck AT, Ward $\mathrm{CH}$, Mendelson $\mathrm{M}$, et al. An inventory for measuring depression. Arch Gen Psychiatry 1961; 4: 561-571.

10. Beck AT, Steer RA, Garbin MG. Psychometric properties of the Beck Depression Inventory: Twenty-five years of evaluation. Clin Psychol Rev 1988; 8: 77-100.

11. Paterson DL, Swindells S, Mohr J, et al. Adherence to protease inhibitor therapy and outcomes in patients with HIV infection. Ann Intern Med 2000; 133: 21-30.

12. Bangsberg DR, Hecht FM, Charlebois ED, et al. Adherence to protease inhibitors, HIV-1 viral load, and development of drug resistance in an indigent population. AIDS 2000; 14: 357-366.

13. Arnsten JH, Demas PA, Farzadegan H, et al. Antiretroviral therapy adherence and viral suppression in HIV-infected drug users: comparison of self-report and electronic monitoring. Clin Infect Dis 2001; 33: 1417-1423.

14. Bangsberg DR, Moss AR, Deeks SG. Paradoxes of adherence and drug resistance to HIV antiretroviral therapy. J Antimicrob Chemother 2004; 53: 696-699.

15. Bangsberg DR, Acosta EP, Gupta R, et al. Adherence-resistance relationships for protease and non-nucleoside reverse transcriptase inhibitors explained by virological fitness. AIDS 2006; 20: 223-231.

16. Skapinakis P, Caldwell D, Hollingworth W, et al. A systematic review of the clinical effectiveness and cost-effectiveness of pharmacological and psychological interventions for the management of obsessive-compulsive disorder in children/adolescents and adults. Health Technol Assess 2016; 20: 1-392.

17. Ye BY, Jiang ZY, Li X, et al. Effectiveness of Cognitive Behavioral Therapy in Treating Bipolar Disorder: An Updated Meta-analysis with Randomized Controlled Trials. Psychiatry Clin Neurosci 2016; doi: 10.1111/pcn.12399.

18. Richards LK, Shingleton RM, Goldman R, et al. Integrative dynamic therapy for bulimia nervosa: An evidence-based case study. Psychotherapy (Chic) 2016; 53: 195-205.

19. Freedland KE, Carney RM, Rich MW, et al. Cognitive Behavior Therapy for Depression and Self-Care in Heart Failure Patients: A Randomized Clinical Trial. JAMA Intern Med 2015; 175: 1773-1782.

20. Harris P, Loveman E, Clegg A, et al. Systematic review of cognitive behavioral therapy for the management of headaches and migraines in adults. Br J Pain 2015; 9: 213-224.

21. Atkinson JH, Grant 1, Kennedy CJ, et al. Prevalence of psychiatric disorders among men infected with HIV. Arch Gen Psychiatris 1988 ; $45: 859-864$.
22. Mulder CL, Emmelkamp PMG, Antoni MH, et al. Cognitive Behavior and experimental group psychotherapy for HIV infected homosexual men: a comparative study. Psychosom Med 1994; 56 423-431.

23. McDaniel JS, Fowlie E, et al. An assessment of psychiatric morbidity and functioning in HIV disease. Gen Hoop Psychiatry 1995; 17: 346-352.

24. Levine AJ, Hinkin $\mathrm{CH}$, Castellon SA, et al. Variations in patterns of highly active antiretroviral therapy (HAART) adherence. AIDS Behav 2005; 9: 355-362.

25. Bergera S, Schada T, von Wylb V, et al. Effects of cognitive behavioral stress management on HIV-1 RNA, CD4 cell counts and psychosocial parameters of HIV-infected persons. AIDS 2008; 22 767-775.

26. Simoni JM, Pearson CR, Pantalone DW, et al. Efficacy of interventions in improving highly active antiretroviral therapy adherence and HIV-1 RNA viral load. A metaanalytic review of randomized controlled trials. J Acquir Immune Defic Syndr 2006; 43 (Suppl 1) S23-S35.

27. Mbuagbaw L, Sivaramalingam B, Navarro T, et al.; Patient Adherence Review (PAR) Team. Interventions for Enhancing Adherence to Antiretroviral Therapy (ART): A Systematic Review of High Quality Studies. AIDS Patient Care STDS 2015; 29: 248-266.

28. Andersen LS, Magidson JF, O'Cleirigh C, et al. A pilot study of a nurse-delivered cognitive behavioral therapy intervention (Ziphamandla) for adherence and depression in HIV in South Africa. J Health Psychol 2016; pii: 1359105316643375.

29. Bere T, Nyamayaro P, Magidson JF, et al. Cultural adaptation of a cognitive behavioural intervention to improve adherence to antiretroviral therapy among people living with HIV/AIDS in Zimbabwe: Nzira Itsva. J Health Psychol 2016; pii: 1359105315626783.

30. Doerfler RE, Goodfellow L. Brief Exposure to Cognitive Behavioral Therapy Reduces Side Effect Symptoms in Patients on Antiretroviral Therapy. J Assoc Nurses AIDS Care 2016; pii: S10553290(16) 000492; doi: 10.1016/j.jana.2016.02.010.

31. Schönnesson LN, Williams ML, Ross MW, et al. Three types of adherence to HIV antiretroviral therapy and their association with AIDS diagnosis and therapy and beliefs about HIV disease. Int J STD AIDS 2007; 18: 369-373.

32. Safren SA, O'Cleirigh CM, Bullis JR, et al.; Cognitive Behavioral Therapy for Adherence and Depression (CBT-AD) in HIV-infected Injection Drug Users: A Randomized Controlled Trial. J Consult Clin Psychol 2012; 80: 404-415.

33. Simoni JM, Wiebe JS, Sauceda JA, et al. A Preliminary RCT of CBT$\mathrm{AD}$ for Adherence and Depression among HIV-Positive Latinos on the U.S. - Mexico Border: The Nuevo Día Study. AIDS Behav 2013 17: 2816-2829. 